\title{
DISPONIBILIDAD DE MATERIAS PRIMAS LÍTICAS SILÍCEAS EN EL EXTREMO SUR DEL MACIZO DEL DESEADO: LOS CASOS DE LA GRUTA Y VIUDA QUENZANA (PROVINCIA DE SANTA CRUZ, ARGENTINA)
}

\author{
NORA FRANCO", PABLO AMBRÚSTOLO** Y NATALIA CIRIGLIANO***
}

\begin{abstract}
RESUMEN
Se presentan aquí los resultados de estudios de disponibilidad de materias primas líticas silíceas en dos espacios del extremo sur del Macizo del Deseado (La Gruta y Viuda Quenzana), en escalas que oscilan entre ca. 1 y $25 \mathrm{~km}$. Los resultados obtenidos refuerzan la idea referida a la existencia de variabilidad en la disponibilidad de rocas en ambas escalas. En general, el rendimiento registrado en las fuentes es alto, siendo sin embargo en Viuda Quenzana más frecuentes las materias primas de calidad excelente y muy buena para la talla, observándose un predominio de calcedonia y otras rocas silíceas. Esta variabilidad en la disponibilidad de rocas silíceas de excelente o muy buena calidad para la talla, sumada a la registrada en la frecuencia de abrigos rocosos (mayor cantidad en el área de Viuda Quenzana que en la de La Gruta), y a la presencia de espejos de agua temporarios que habrían variado en relación con las lluvias a lo largo del tiempo, debieron haber tenido importancia en las estrategias seguidas por los cazadores-recolectores.
\end{abstract}

PALABRAS CLAVE: Patagonia, rocas silíceas, disponibilidad, variabilidad, escalas.

SILICEOUS ROCKS AVAILABILITY IN THE SOUTHERN END OF THE DESEADO MASSIF: LA GRUTA AND VIUDA QUENZANA (PROVINCE OF SANTA CRUZ, ARGENTINA)

\section{ABSTRACT}

Results of studies of siliceous rock availability in two areas of the southern end of the Deseado Massif (La Gruta and Viuda Quenzana) are presented. Variability is compared in scales ranging from ca. 1 to $25 \mathrm{~km}$. The results point to the existence of variability at both scales.

The recorded performance is high in both spaces, but siliceous raw materials of excellent and very good quality are more frequent in Viuda Quenzana, where chalcedony and other siliceus rocks are the

IMHICIHU (CONICET) y UNIVERSIDAD DE BUENOS AIRES - Saavedra 15, 5to. Piso, Capital (C.P. 1084), Buenos Aires, Argentina, e-mail: nvfranco2008@gmail.com.

* CONICET (Departamento Científico de Arqueología, Museo de La Plata, UNLP), Paseo del Bosque s/n, La Plata (C.P. 1900), Argentina, e-mail: pambrustolo@hotmail.com.

CONICET (IMHICIHU). Saavedra 15, 5to. piso, Capital (C.P. 1083), Argentina, e-mail: naticirigliano@hotmail.com. 
most frequent ones. This variability in siliceous rock availability coupled with the frequency of rock shelters and caves (more abundant in Viuda Quenzana than in La Gruta), and the presence of temporary water bodies which have probably varied in relation to rainfall over time must have been important for huntergatherers strategies.

KEY WORDS: Patagonia, siliceous rocks, availability, variability, scales.

\section{INTRODUCCIÓN}

Los recursos líticos se encuentran disponibles en general en concentraciones localizadas $e$ involucran costos de búsqueda y aprovisionamiento (cf. por ejemplo Bousman 1993; Hayden 1989). El Macizo del Deseado presenta un caso particular, ya que en general la oferta de rocas silíceas es alta (cf. por ejemplo, Cattáneo 2004; Echeveste 2005; Hermo 2008; Mansur-Franchomme 1984; Panza 1994; Skarbun 2009). Sin embargo, en la zona central del Macizo se ha registrado variabilidad en la disponibilidad (cf. por ejemplo Hermo 2008). Nos interesa en este trabajo dar cuenta de la existencia de variaciones en la disponibilidad de materias primas silíceas en el extremo sur del Macizo del Deseado entre dos diferentes sectores, localizados a $25 \mathrm{~km}$ entre sí. Asimismo, comenzamos a explorar la variabilidad intra sector.
Entendemos que la comprensión de estas variaciones, unidas a las existentes en la oferta de reparos rocosos y agua es esencial para entender la forma y tempo de exploración de estos espacios, así como las razones de selección y forma de utilización de algunos de ellos durante la ocupación efectiva (sensu Borrero 1989-90, 1994-95). Este tema es especialmente relevante si se toma en cuenta que en el extremo sur del Macizo del Deseado se han recuperado evidencias de presencia humana comprendidas entre ca. 10.840 (Franco et al. 2010a) y ca. 400 años AP (Franco et al. 2011). En ese marco, el objetivo de este trabajo es evaluar las variaciones en la disponibilidad y productividad de materias primas líticas en dos sectores del sur del Macizo del Deseado: Viuda Quenzana y La Gruta (Fig. 1).

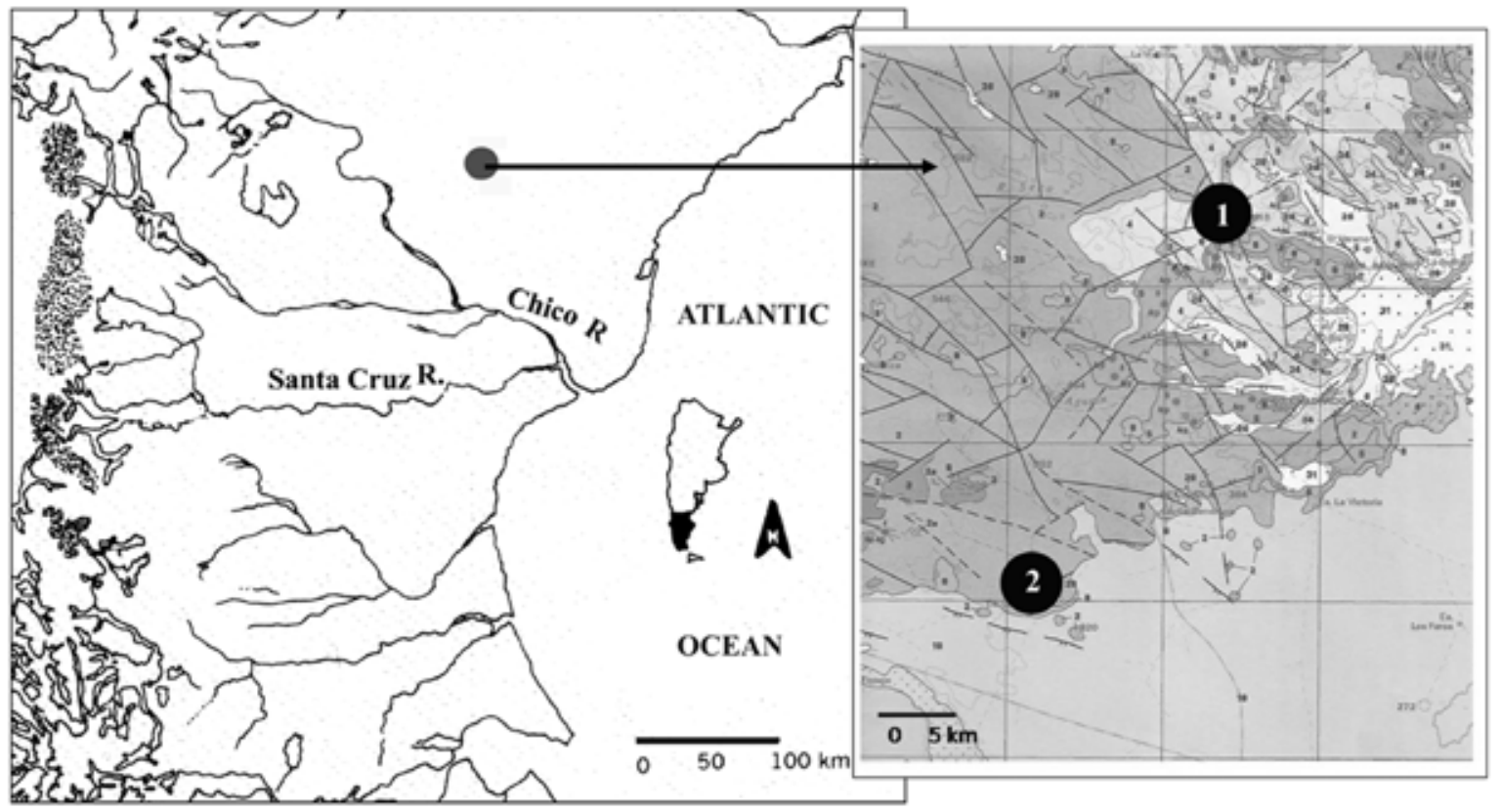

Fig. 1. Localización de los espacios muestreados. Referencias: 1. Viuda Quenzana, 2. La Gruta. 


\section{ANTECEDENTES Y DESCRIPCIÓN DEL ÁREA}

La bibliografía geológica para el área del Macizo indica la presencia frecuente de rocas de muy buena y/o excelente calidad para la talla. Se hace referencia a la existencia de ignimbritas, maderas silicificadas, tobas y filones epitermales de cuarzo, con algunos sectores muy silicificados con calcedonia y jaspe (entre otros, Echavarría 2004; Echeveste 2005; Giacosa et al. 1998; Panza 1994). Como ya mencionamos, esta disponibilidad no es homogénea. Estudios recientes sugieren que la disponibilidad de rocas de calidad muy buena o excelente para la talla es mayor al nordeste de nuestra área de estudio (Franco et al. 2012), tanto en fuentes primarias como secundarias de aprovisionamiento lítico (cf. Luedtke 1979; Nami 1992).

Este trabajo se centraliza en el sur del Macizo del Deseado, en dos espacios separados por una distancia de ca. 25 km: Viuda Quenzana y La Gruta (Fig. 1). En cada uno de ellos, los trabajos arqueológicos abarcaron una superficie aproximada de $10 \mathrm{~km}$ por $10 \mathrm{~km}$. Viuda Quenzana posee cotas entre ca. 350 y $400 \mathrm{~m}$, mientras que La Gruta tiene alturas entre ca. 300 y $350 \mathrm{msnm}$. El primero de los sectores presenta afloramientos rocosos de toba e ignimbritas (formaciones Chon Aike y Bajo Grande), mientras que La Gruta presenta ignimbritas (Formación Chon Aike) y afloramientos correspondientes a la Formación Monte León, compuestos por areniscas coquinoideas y coquinas (Panza y Marín 1998). Por otra parte, en proximidades de este último sector, la bibliografía indica la presencia de bancos interestratificados de tobas finas con niveles silíceos de lo que se denominan sinters, que en algunos sectores pueden estar fuertemente silicificados y pertenecen a la formación La Matilde (Schalamuk et al. 2002). Geólogos locales han mencionado también la presencia de sinters en el área de La Gruta (Kain com. pers. 2012). Por otra parte, en cuanto a la geomorfología, en el espacio relevado en Viuda Quenzana se registró la presencia de cañadones y bajos lagunares, mientras que en La Gruta se han identificado sólo estos últimos.

\section{METODOLOGÍA}

Se utilizó la metodología de relevamiento de fuentes potenciales de aprovisionamiento lítico diseñada por Franco y Borrero (1999), a la que se agregaron otras variables de análisis: ancho y espesor de los nódulos (sensu Aschero 1983), forma de presentación (bloques o guijarros), rendimiento y productividad.

Se considera al rendimiento como el total de rocas aptas para la talla recuperables en un determinado tiempo de recolección. En este trabajo, el mismo se definió como alto cuando dos personas obtuvieron 10 ó más rocas aptas para la talla en 10 minutos de búsqueda, incluyéndose dentro de éstas a las de calidades buenas, muy buenas y excelentes (sensu Aragón y Franco 1997).

Por otra parte, se entiende como productividad al porcentaje de materia prima aprovechable en el nódulo, evaluado en relación con la presencia de impurezas, planos de debilidad y grado de homogeneidad en la silicificación (cf. Franco et al. 2012). A los fines de este trabajo, se considera productividad alta cuando dicho porcentaje de materia prima oscila entre un 80 y 100\% de la misma calidad. Ésta se caracteriza como regular cuando el porcentaje oscila entre 50 y 79,9\%, baja entre un 20 y 49,9\% y muy baja entre 0,1 y $19,9 \%$. Se consideran como aprovechables las materias primas de calidades regular a excelente para la talla.

Se analizó la variabilidad presente a una escala de $25 \mathrm{~km}$-la existente entre La Gruta y Viuda Quenzana- y a una escala menor, analizando la variación dentro de cada uno de esos espacios. Por este motivo, se tomó la información procedente de dos muestreos en cada uno de ellos (Viuda Quenzana y La Gruta). La información utilizada en este trabajo se refirió a: tipo y porcentaje de materia prima, calidad para la talla (sensu Aragón y Franco 1997), forma de presentación (rodado o bloque), dimensiones mayores, productividad y rendimiento.

\section{RESULTADOS}

Se detallan a continuación los resultados obtenidos en cada uno de los espacios analizados.

\section{a) Viuda Quenzana}

Se relevaron dos sectores con características geomorfológicas diferentes (Fig. 2 y 3). En cada uno de ellos se efectuó un muestreo. Se trata del cañadón del Río Seco -emplazado en la Formación Chon Aike (Panza y Marín 1998)- y un sector próximo a 


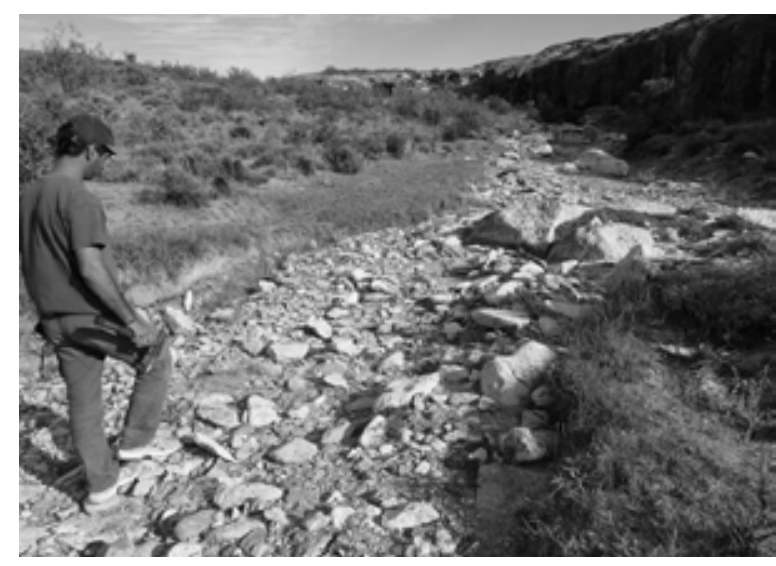

Fig. 2. Vista del cañadón del Río Seco (Viuda Quenzana).

Tabla 1. Rendimiento (2 personas, 10 minutos)

\begin{tabular}{|l|c|}
\hline \multicolumn{1}{|c|}{ Espacio muestreado } & Rendimiento \\
\hline Viuda Quenzana, Río Seco & 12 \\
\hline Viuda Quenzana, La Barda & 13 \\
\hline La Gruta, Laguna 1 & 7 \\
\hline La Gruta, Laguna 2 & 18 \\
\hline
\end{tabular}

un afloramiento de toba al sudeste de este cañadón, denominado La Barda -correspondiente a la Formación Bajo Grande (Panza y Marín 1998)-.

El rendimiento registrado, en los dos casos, es alto (Tabla 1). Si bien en ambos sectores se identificaron rocas silíceas, en La Barda predominan las

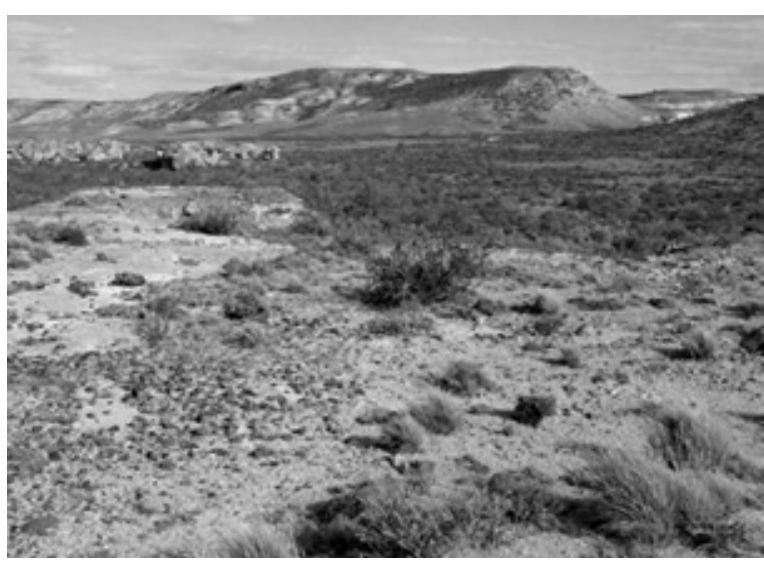

Fig. 3. Vista del sector de La Barda (Viuda Quenzana).

calcedonias, mientras que en el río Seco lo hacen otras rocas silíceas (ver Tabla 2). Tanto en el río Seco como en la Barda la mayor frecuencia corresponde a rocas de muy buena calidad para la talla, existiendo también en menor medida ejemplares de excelente y buena calidad. En lo que se refiere a las materias primas de inferior calidad, éstas son más frecuentes en el Río Seco que en La Barda (Tabla 3).

Los dos sectores muestreados corresponden a fuentes potenciales de aprovisionamiento secundarias, en los que predominan los bloques en relación con los guijarros (ver Tabla 4, Fig. 4 y 5). El porcentaje de los bloques es mayor en el Río

Tabla 2. Tipos de materias primas. Ref: Mad. sil: madera silicificada; Rocas sil: otras rocas silicificadas.

\begin{tabular}{|l|c|c|c|c|c|c|c|}
\hline Materias primas & Calcedonia & Madera sil. & Rocas sil. & Dacita & Riolita & Indet. & Total \% \\
\hline Viuda Quenzana, La Barda & $71,43 \%$ & $0,00 \%$ & $28,57 \%$ & $0,00 \%$ & $0,00 \%$ & $0,00 \%$ & $100 \%$ \\
\hline Viuda Quenzana, Río Seco & $11,76 \%$ & $0,00 \%$ & $88,24 \%$ & $0,00 \%$ & $0,00 \%$ & $0,00 \%$ & $100 \%$ \\
\hline La Gruta, Laguna 1 & $9,09 \%$ & $9,09 \%$ & $63,64 \%$ & $0,00 \%$ & $18,18 \%$ & $0,00 \%$ & $100 \%$ \\
\hline La Gruta, Laguna 2 & $40,85 \%$ & $0,00 \%$ & $35,20 \%$ & $1,41 \%$ & $21,13 \%$ & $1,41 \%$ & $100 \%$ \\
\hline
\end{tabular}

Tabla 3. Calidades para la talla.

\begin{tabular}{|l|c|c|c|c|c|c|}
\hline \multicolumn{1}{|c|}{ Calidad para la talla } & Excelente & Muy buena & Buena & Regular & Mala & Total \\
\hline Viuda Quenzana, La Barda & $7,14 \%$ & $64,29 \%$ & $21,43 \%$ & $7,14 \%$ & $0,00 \%$ & $100 \%$ \\
\hline Viuda Quenzana, Río Seco & $5,88 \%$ & $41,18 \%$ & $23,53 \%$ & $23,53 \%$ & $5,88 \%$ & $100 \%$ \\
\hline La Gruta, Laguna 1 & $4,55 \%$ & $0,00 \%$ & $27,27 \%$ & $50,00 \%$ & $18,18 \%$ & $100 \%$ \\
\hline La Gruta, Laguna 2 & $1,41 \%$ & $29,58 \%$ & $33,80 \%$ & $21,13 \%$ & $14,08 \%$ & $100 \%$ \\
\hline
\end{tabular}

Tabla 4. Caracteres básicos (sensu Aschero 1983) de las rocas.

\begin{tabular}{|l|c|c|c|}
\hline \multicolumn{1}{|c|}{ Caracteres básicos } & Bloque & Guijarro & Total \\
\hline Viuda Quenzana, La Barda & $57,14 \%$ & $42,86 \%$ & $100 \%$ \\
\hline Viuda Quenzana, Río Seco & $82,35 \%$ & $17,65 \%$ & $100 \%$ \\
\hline La Gruta, Laguna 1 & $100 \%$ & $0,00 \%$ & $100 \%$ \\
\hline La Gruta, Laguna 2 & $100 \%$ & $0,00 \%$ & $100 \%$ \\
\hline
\end{tabular}




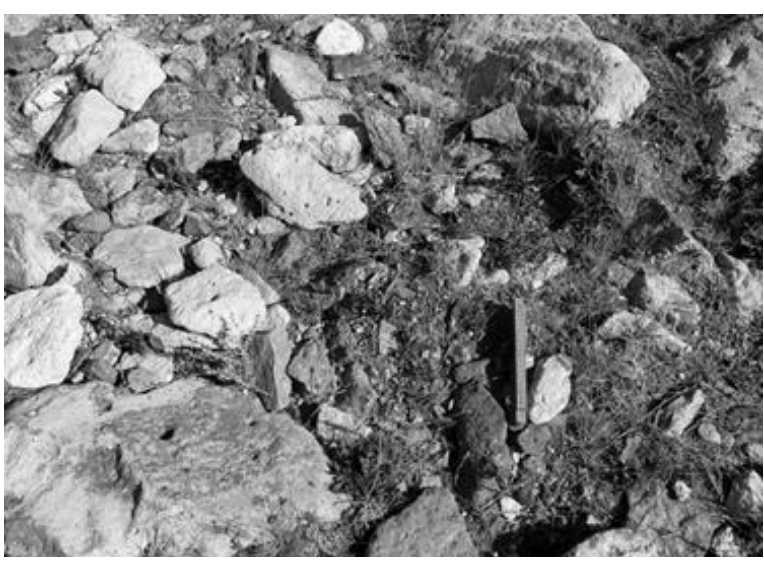

Fig. 4. Detalle de la forma de presentación de rocas en un sector del cañadón del Río Seco (Viuda Quenzana).

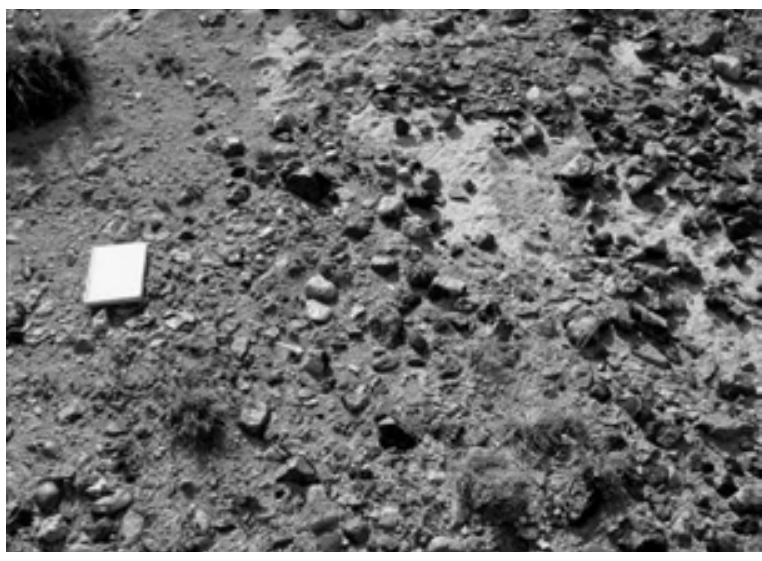

Fig. 5. Detalle de la forma de presentación de rocas en un sector de La Barda (Viuda Quenzana).

Tabla 5. Tamaños recuperados. Referencias: Viuda Q: Viuda Quenzana.

\begin{tabular}{|l|c|c|c|c|}
\hline \multicolumn{1}{|c|}{ Tamaños (en mm) } & Viuda Q., La Barda & Viuda Q, Río Seco & La Gruta, Laguna 1 & La Gruta, Laguna 2 \\
\hline Media & 48,93 & 134,12 & 81,59 & 97,69 \\
\hline Desvío estándar & 11,89 & 40,94 & 28,25 & 33,15 \\
\hline
\end{tabular}

Tabla 6. Productividad. Referencia: no corr: no corresponde.

\begin{tabular}{|l|c|c|c|c|c|}
\hline \multicolumn{1}{|c|}{ Productividad } & Alta & Media & Baja & Muy baja & No corr. \\
\hline Viuda Quenzana, La Barda & $100 \%$ & $0,00 \%$ & $0,00 \%$ & $0,00 \%$ & $0,00 \%$ \\
\hline Viuda Quenzana, Río Seco & $64,71 \%$ & $11,76 \%$ & $17,65 \%$ & $0,00 \%$ & $5,88 \%$ \\
\hline La Gruta, laguna 1 & $66,67 \%$ & $14,29 \%$ & $4,76 \%$ & $0,00 \%$ & $14,29 \%$ \\
\hline La Gruta, laguna 2 & $46,48 \%$ & $30,99 \%$ & $7,04 \%$ & $1,41 \%$ & $14,08 \%$ \\
\hline
\end{tabular}

Seco $(82,35 \%$ versus $57,14 \%$ de la muestra en el caso de La Barda) (Tabla 4), en donde se registraron también las mayores dimensiones y la mayor variabilidad en tamaños (Tabla 5). La productividad más alta, en cambio, se registra en las muestras de La Barda (Tabla 6).

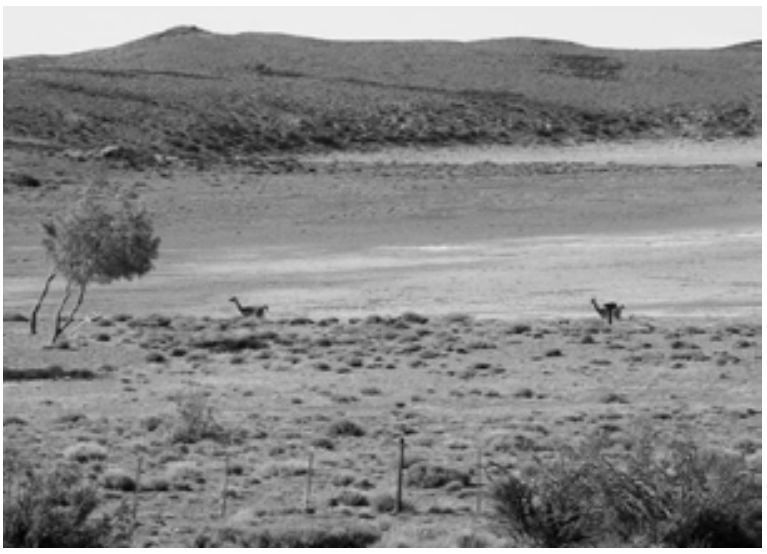

Fig. 6. Vista parcial de la Laguna 1 (La Gruta). b) La Gruta

Se relevaron dos bajos lagunares separados por una distancia de ca. 1,2 km (Fig. 6 y 7), denominados Laguna 1 y 2 (cf. Franco y Cattáneo MS; Franco et al. 2010b), tomándose la información

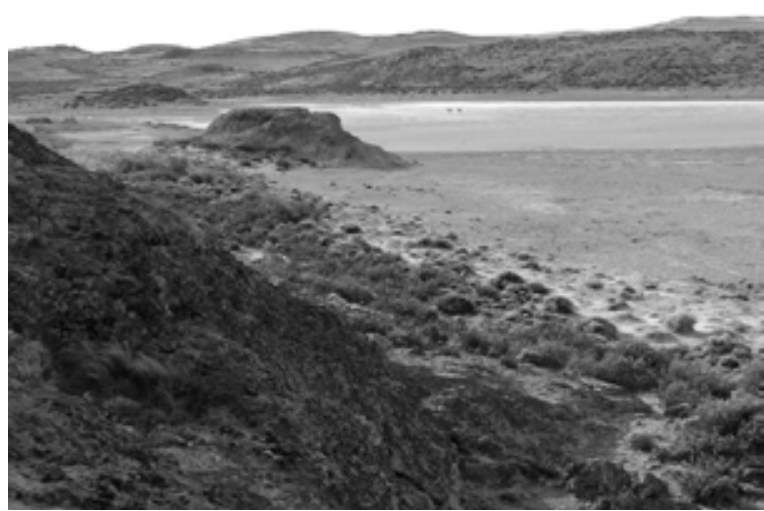

Fig. 7. Vista parcial de la Laguna 2 (La Gruta). 


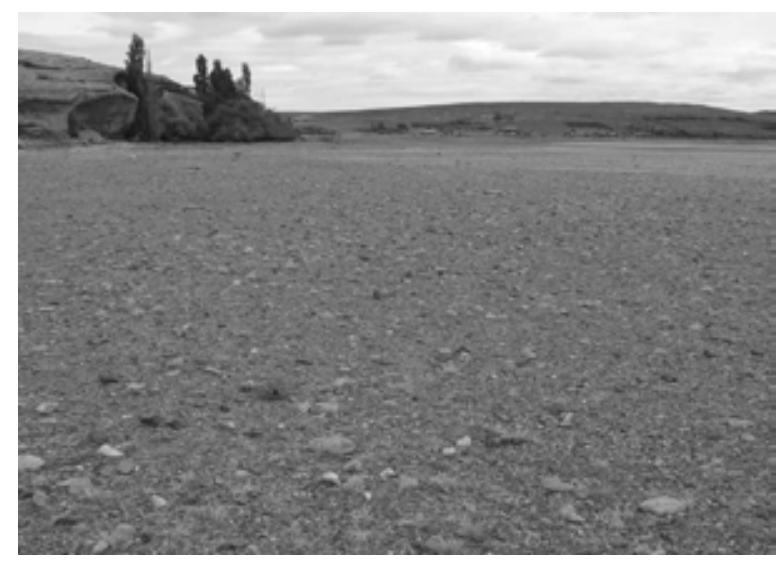

Fig. 8. Vista de la forma de presentación de materias primas en la Laguna 1 (La Gruta).

de un muestreo en cada uno de ellos (Fig. 8 y 9). En ambos sectores los muestreos se realizaron en la porción sur, correspondiendo los relevamientos de la Laguna 1 al interior de la misma, mientras que los de la Laguna 2 se hicieron en lomadas próximas a ella.

El muestreo de la Laguna 2 presenta los rendimientos más altos (Tabla 1). Si bien el porcentaje de rocas silíceas es similar en ambos sectores muestreados, en ésta las calcedonias son más abundantes (Tabla 2). En la Laguna 2 predominan los ejemplares de calidad buena para la talla, siguiéndoles los de muy buena calidad, mientras que en la Laguna 1 las rocas regulares son las más abundantes, seguidas por las de calidad buena (Tabla 3). Las materias primas de calidad mala son más frecuentes también en La Laguna 1.

Cabe señalar que, si bien en el caso de la Laguna 2 se trata de una fuente primaria, no se conoce aún el lugar exacto de procedencia de las rocas recuperadas en la Laguna 1 , aunque se entiende. Se trata de un depósito secundario en el que se recuperaron ejemplares procedentes de vetas. En todos los casos, se trata de bloques (Tabla 4). Las mayores dimensiones corresponden a las rocas registradas en la Laguna 2 (Tabla 5). Ésta es la muestra que presenta la mayor variabilidad en tamaños (Tabla 5). La productividad más alta, en cambio, se registra en las muestras de la Laguna 1 (Tabla 6).

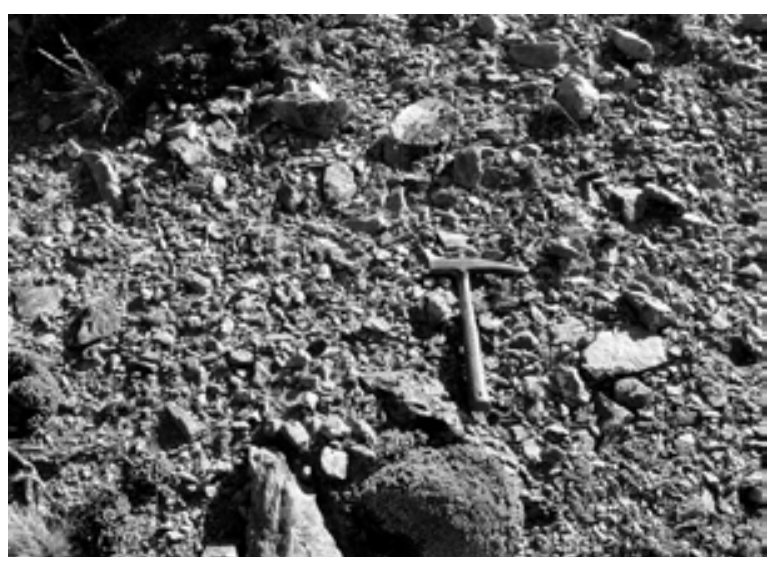

Fig. 9. Vista de la forma de presentación de materias primas en la Laguna 2 (La Gruta).

\section{COMPARACIÓN ENTRE VIUDA QUENZANA Y LA GRUTA}

Los resultados obtenidos refuerzan la idea planteada previamente referida a la existencia de variabilidad en la disponibilidad de rocas en el sur del Macizo del Deseado, evaluada a una escala mayor que la analizada aquí (Franco et al. 2012). En este caso, la variabilidad se ha registrado tanto a escalas de ca. $25 \mathrm{~km}$ como de ca. $1 \mathrm{~km}$. Como ya se mencionó en el acápite anterior, se han encontrado diferencias entre el cañadón del Río Seco y La Barda, en Viuda Quenzana, y entre las dos lagunas de La Gruta.

En general, el rendimiento registrado es alto (Tabla 1). Sin embargo, en Viuda Quenzana son más frecuentes las materias primas de calidad excelente y muy buena para la talla (Tabla 3). Como puede observarse en la Tabla 3, mientras en Viuda Quenzana las rocas de calidad muy buena y excelente superan el $45 \%(71,43 \%$ y $47,06 \%$ de la muestra respectivamente), en La Gruta sólo alcanzan el 31\% de la muestra (4,55\% y 30,99\% respectivamente). En contraposición, las rocas de calidad regular y mala son más frecuentes en el área de La Gruta. Los ejemplares que presentan buena calidad para la talla se encuentran en frecuencias similares en ambos sectores del espacio.

De manera general podemos decir también que, si bien en ambos espacios muestreados predominan las calcedonias y otras rocas silíceas, en Viuda Quenzana su disponibilidad es mayor, alcanzando 
el $100 \%$ de la muestra recolectada (Tabla 2). La dacita y la riolita están representadas únicamente en La Gruta (Tabla 2). Este hecho podría deberse a una cuestión de muestreo, debido a que dentro de cada uno de los espacios se seleccionaron las rocas de mejor calidad para la talla. Por otra parte, en ambas áreas se han recogido bloques, limitándose los guijarros a Viuda Quenzana (Tabla 4). La productividad de los nódulos es alta en ambos sectores del espacio, registrándose la existencia de variaciones dentro de los espacios muestreados (Tabla 6). Cabe señalar que en el área de Viuda Quenzana, muestreo La Barda, la misma alcanza el 100\%. Las mayores dimensiones corresponden al cañadón del Río Seco, en Viuda Quenzana (Tabla 2). El otro muestreo realizado, en cambio, presenta las dimensiones más pequeñas de la totalidad de las muestras recogidas (La Barda, cf. Tabla 2).

\section{CONSIDERACIONES GENERALES}

Los resultados obtenidos apuntan a la existencia de variabilidad en la disponibilidad de rocas en el extremo sur del Macizo del Deseado. Si bien las materias primas de calidad excelente para la talla están presentes, no son ubicuas. Estas variaciones habían sido registradas más hacia el norte del Macizo (cf. por ejemplo, Hermo 2008), observándose también diferencias en la calidad y productividad de las materias primas entre distintos sectores del este y sur del mismo (Franco et al. 2012). Las poblaciones que utilizaron el extremo sur del Macizo en el pasado hicieron uso de los recursos líticos disponibles teniendo en cuenta las variaciones existentes, una vez que éstas fueron conocidas. El reconocimiento de las mismas, por otra parte, debió afectar también el tempo de exploración e incorporación de estos espacios. En general, puede observarse la utilización de rocas de excelente calidad desde los momentos tempranos del poblamiento del sur del Macizo (cf. Franco et al. 2010b). Además, desde los momentos iniciales de presencia humana en este espacio se observa el transporte y descarte de obsidiana negra, disponible a ca. $150 \mathrm{~km}$ (Franco et al. 2010a), probablemente como parte del equipamiento personal (cf. Civalero y Franco 2003).

La variabilidad existente en la disponibilidad de rocas silíceas de excelente y muy buena calidad para la talla, sumada a la registrada en la frecuen- cia de abrigos rocosos (mayor disponibilidad en el área de Viuda Quenzana que en la de La Gruta), y a la presencia de espejos de agua temporarios que habrían variado en relación con las lluvias a lo largo del tiempo (cf. Mancini et al. 2011), debieron haber tenido importancia en las estrategias seguidas por los cazadores-recolectores. Entendemos que el conocimiento en las variaciones en la disponibilidad de rocas silíceas dentro del Macizo, tanto en las escalas analizadas aquí como en escalas mayores, sumada a la ampliación de prospecciones y excavaciones, contribuirán a comprender las elecciones efectuadas por los cazadores-recolectores y a obtener una imagen más dinámica de la forma de poblamiento y utilización de este sector del espacio.

\section{AGRADECIMIENTOS}

Este trabajo se llevó a cabo en el marco de los proyectos PIP (CONICET) 0356 y UBACyT (Universidad de Buenos Aires) 01/W404. Queremos expresar nuestro agradecimiento a la Minera Triton y, en particular, a Mathew Andrews, Carlos Baetti y Sergio Kain, por la colaboración recibida. A la Minera Piedra Grande y en particular, a Claudio Iglesias y Carlos Parretti. A Roberto Ulloa y Luciana Frank. A Eugenio Aragón (CIC-CONICET) por las determinaciones de materias primas, a la Dirección de Patrimonio de la provincia de Santa Cruz y a la Dirección de Cultura de Gobernador Gregores. En particular, a Marcelo Cebeira y Liliana Costas. A los dos evaluadores anónimos, cuyas correcciones contribuyeron a mejorar el manuscrito.

\section{BIBLIOGRAFÍA}

ASCHERO, C. A. 1983. Revisión del ensayo para una clasificación morfológica de artefactos líticos aplicada a estudios tipológicos comparativos. Informe presentado al CONICET. MS.

ARAGÓN, E. Y FRANCO, N. V. 1997. Características de rocas para la talla por percusión y propiedades petrográficas. Anales del Instituto de la Patagonia. Serie Ciencias Humanas 25:187-199.

BORRERO, L. A. 1989-90. Evolución cultural divergente en la Patagonia Austral. Anales del Instituto de la Patagonia. Serie Ciencias Sociales 19:133-139.

BORRERO, L. A. 1994-95. Arqueología de la Patagonia. Palimpsesto. Revista de Arqueología 4: 9-69. 
BOUSMAN, C.B. 1993. Hunter-Gatherer Adaptations, Economic Risk and Tool Design. Lithic Technology 18 (1/2): 59-86.

CATTÁNEO, G. R. 2004. Desarrollo metodológico para el estudio de fuentes de aprovisionamiento lítico en la meseta central santacruceña, Patagonia Argentina. Estudios Atacameños, 28: 105-119.

CIVALERO, M. T. y N. V. FRANCO. 2003. Early Human Occupations in Western Santa Cruz Province, Southernmost South America. Quaternary International 109-110: 77-86.

ECHAVARRIA, L. E. 2004. Los fluidos hidrotermales formadores de la mineralización epitermal el Dorado-Monserrat, Macizo del Deseado. Revista de la Asociación Geológica Argentina, 59(1):70-82.

ECHEVESTE, H. 2005. Travertinos y jasperoides de Manantial Espejo, un ambiente Hot Spring Jurásico. Macizo del Deseado, Provincia de Santa Cruz, Argentina. Latin American Journal Sedimentoloy and Basin Analysis 12(1):33-48.

FRANCO, N. V.; AMBRÚSTOLO, P.; MARTUCCI, M.; BROOK, G.; MANCINI, M. V. Y CIRIGLIANO, N. 2010a. Early human occupation in the southern part of the Deseado Massif (Patagonia, Argentina). Current Research in the Pleistocene, 7: 13-16.

FRANCO, N. V. Y BORRERO, L. A. 1999. Metodología de análisis de la estructura regional de recursos líticos. En: Aschero, C. A.; Korstanje, M. A. y Vuoto, P. M. En los Tres Reinos: Prácticas de Recolección en el Cono Sur de América. pp. 27-37. Ediciones Magna Publicaciones. Tucumán.

FRANCO, N. V. Y R. CATTANEO. Trabajos arqueológicos en el área de La Gruta (Gobernador Gregores, Santa Cruz, Argentina). MS.

FRANCO, N. V., M. MARTUCCI, P. AMBRÚSTOLO, G BROOK, M. V. MANCINI Y N. CIRIGLIANO. 2010b. Ocupaciones humanas correspondientes a la transición Pleistoceno- Holoceno al sur del macizo del deseado: El área de La Gruta (Provincia de Santa Cruz, Argentina). Relaciones de la Sociedad Argentina de Antropología XXXV: 301-308.

FRANCO, N. V., P. AMBRÚSTOLO, A. ACEVEDO, N. CIRIGLIANO Y M. VOMMARO. 2011. Prospecciones en el sur del Macizo del Deseado. Los casos de La Gruta y Viuda Quenzana. Poster presentado en las VIII Jornadas de Arqueología de la Patagonia. Malargüe, Mendoza.

FRANCO, N. V., P. AMBRÚSTOLO, F. SKARBUN, N. CIRIGLIANO Y M. MARTUCCI. 2012. El Macizo del Deseado como fuente de aprovisionamiento de rocas silíceas. Variaciones en la disponibilidad y circulación: algunos ejemplos. En prensa en Cazadores Recolectores del Cono Sur.
GIACOSA, R. E. ; CÉSARI, O. Y GENINI, A. 1998. Descripción de la Hoja geológica 4766 III y IV "Puerto Deseado", provincia de Santa Cruz. Servicio Geológico Minero Argentino. Instituto de Geología y Recursos Minerales. Boletín $N^{\circ} 213$. Buenos Aires.

HAYDEN, B. 1989. From the chopper to the celt: the evolution of resharpening techniques. En: Time, energy and stone tools, ed. R. Torrence, pp. 7-16. Cambridge University Press. Cambridge.

HERMO, D. 2008. Los cambios en la circulación de materias primas líticas en ambientes mesetarios de la Patagonia. Una aproximación para la construcciòn de paisajes arqueológicos de las sociedades cazadoras-recolectoras. Tesis doctoral inédita. Universidad de La Plata. MS.

LUEDTKE, B. E. 1979. The Identification of Sources of Chert Artifacts. American Antiquity, 44:744-756.

MANCINI, M. V., F. P. BAMONTE, N. V. FRANCO Y G. BROOK. 2012. Paleoenvironmental conditions and changing early occupations of the south of the Deseado Massif (Patagonia, Argentina). Poster presentado al XVIII Congreso Internacional del INQUA. Berna.

MANSUR-FRANCHOMME, M. E. 1984. Préhistoire de la Patagonie. Lindustrie "Nivel 11" de la province de Santa Cruz (Argentine). Technologie lithique et traces d'utilisation. BAR International Series 216. Oxford.

NAMI, H. 1992. El subsistema tecnológico de la confección de instrumentos líticos y la explotación de los recursos del ambiente: una nueva guía de aproximación. Shincal, 2:33-53.

PANZA, J. L. 1994. Descripción de la Hoja Geológica 4969-II. Tres Cerros Escala 1:250.000. Provincia de Santa Cruz. Servicio Geológico Nacional. Buenos Aires.

PANZA, J. L. Y MARÍN, G. 1998. Hoja Geológica 4969-I Gobernador Gregores, Provincia de Santa Cruz. Programa Nacional de Cartas Geológicas de la República Argentina 1:250.000. Boletín $\mathrm{N}^{\circ}$ 239. Secretaría de Minería de la Nación. Dirección Nacional del Servicio Geológico. Buenos Aires.

SCHALAMUK, I. B., R. E. de BARRIO, M. A. ZUBIA, A. GENINI y J. VALVANO. 2002. Mineralizaciones auro-argentíferas del Macizo del Deseado y su ecuadre metalogénico. En M. J. Haller (Ed). Geología y Recursos naturales de Santa Cruz. Relatorio del XV Congreso Geológico Argentino. El Calafate IV-2: 679-713.

SKARBUN, F. 2009. La organización tecnológica en grupos cazadores recolectores desde las ocupaciones del Pleistoceno final al Holoceno tardío, en la Meseta Central de Santa Cruz. Tesis Doctoral. Facultad de Ciencias Naturales y Museo. Universidad Nacional de La Plata. MS. 